\title{
IVD.I9 - Standardization of quantitative real time PCR for differential diagnostic of Parvovirus B 19 infection in acute liver failure patients
}

\author{
Arthur Daniel Rocha Alves ${ }^{1 *}$; Barbara Barbosa Langella ${ }^{1}$; Rita de Cassia Nasser Cubel Garcia ${ }^{2}$; \\ Oswaldo Gonçalves Cruz ${ }^{3}$; Marcelo Alves Pinto ${ }^{1}$; Luciane Almeida Amado Leon ${ }^{1}$. \\ 1Fiocruz/IOC; \\ 2UFF - Universidade Federal Fluminense; \\ 3Fiocruz - Fundação Oswaldo Cruz.
}

Introduction: Human Parvovirus B19 (B19V) is a common pathogen worldwide. This infection is usually acute and self-limited and may cause a wide variety of clinical manifestations. B19V has been recognized as a self-limiting cause of acute hepatitis and as uncommon cause of ALF in children and adults. A high prevalence of B19V-DNA has been associated with livers of patients with liver disease, being detectable for years after acute infection, which suggests a persistent B19V-infection in liver.

Objective: To standardize and validate a real-time PCR (qPCR) for detection and quantification of B19V-DNA, in order to establish a differential diagnosis for B19V infection in ALF patients.

Methodology: The qPCR techniques were based on Sybr Green ${ }^{\circledR}$ and TaqMan ${ }^{\circledast}$ methodologies. To evaluate the quality parameters of both methods, serum samples from patients with $(n=33)$ and without $(n=38)$ B19V infection were used. The absolute quantification was determined by a synthetic standard curve based on NS1 region of B19V. In order to exclude the possibility of false negative qPCR results due to PCR inhibitors, it was used a reference control (TaqMan ${ }^{\circledast}$ RNAse $\mathrm{P}$ ). The performance of the assays was evaluated testing archived serum and hepatic tissue explants from 10 patients with cryptogenic ALF.

Results: The Sybr Green methodology showed 97\% of efficiency, the limits of detection and quantification were 62.6 and 53200 copies $/ \mathrm{mL}$, respectively. The Taqman methodology showed $95 \%$ of efficiency, the limits of detection and quantification were 4.48 and 310 copies $/ \mathrm{mL}$, respectively. Sybr green showed $97.3 \%$ of specificity ( $\mathrm{p}<0.001,95 \% \mathrm{CI} 0.86$ to 0.99 ), and the Taqman showed $100 \%$ of specificity ( $\mathrm{p}<0.001,95 \%$ CI 0.90 to 1.00 ). Both qPCR methodologies showed sensibilities of $100 \%$ ( $\mathrm{p}<0.001,95 \% \mathrm{CI} 0.89$ to 1.00 ). No cross-reaction was observed by Taqman methodology, when testing other viruses. However, a B19V false-positive result was observed using Sybr Green methodology, in a Dengue positive sample. Despite a small variation for the RNase P Cts observed between serum (31.12 to 35.22) and hepatic tissues (16.01 to 25.44), through this analysis, there were no false negative results, supporting the accuracy of the results. Among ten patients with cryptogenic ALF, three (30\%) were positive for B19V DNA in serum and hepatic tissue explant through standardized qPCR. All of them were IgG anti-B19V positive and none of them were IgM anti-B19V. The mean viral load in

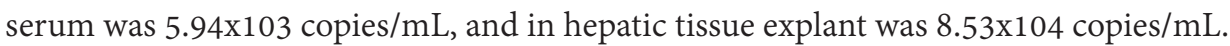

Conclusion: These results demonstrate the importance of using a sensible and specific molecular method to clarify the B19V infection in cryptogenic ALF cases; using serum and hepatic tissue explant samples. The assay showed optimal performance characteristic and demonstrated to be fit for differential diagnosis and investigation of ALF causes.

Keywords: Acute Liver Failure; Human Parvovirus B19; Real-time quantitative PCR 